\title{
Ambliopia por estrabismo: estudo retrospectivo de pacientes em hospital universitário
}

\author{
Strabismic amblyopia: a retrospective study on patients from a \\ universityhospital
}

\author{
Deborah Salerno Costa ${ }^{1}$ \\ Rita de Cássia Andrade Klein ${ }^{2}$ \\ Cristiane de Almeida Leite ${ }^{3}$ \\ Maria Antonieta da Anunciação Ginguerra ${ }^{4}$ \\ Mariza Polati5
}

\begin{tabular}{l} 
RESUMO \\
\hline Objetivo: Avaliar a influência da adesão ao tratamento, da gravidade da \\
ambliopia e da idade de início do tratamento em pacientes com ambliopia \\
por estrabismo submetidos à terapia oclusiva. Métodos: Analisaram-se \\
569 prontuários de pacientes com ambliopia por estrabismo atendidos no \\
Hospital das Clínicas da Faculdade de Medicina da USP no período de \\
1983 a 2000. Os critérios de exclusão foram: perda de seguimento, idade \\
maior que 12 anos, presença de nistagmo e outras doenças oculares. \\
Todos foram submetidos a exame oftalmológico completo com avaliação \\
da motilidade ocular, divididos por faixas etárias e classificados quanto \\
ao tipo de estrabismo, gravidade da ambliopia e adesão ao tratamento. \\
Os dados foram analisados estatisticamente pelo método de Fisher. \\
Resultados: Foramincluídos 198 pacientes (34,8\%). Nãohouve diferença \\
de adesão nos diversos grupos etários. A taxa de sucesso foi maior nos \\
pacientes com boa adesão independente da gravidade da ambliopia. \\
Porém a adesão ao tratamento foi menor no grupo com ambliopia grave, \\
que foi o mais freqüente e obteve menor taxa de sucesso em nossa \\
amostra. Não houve relação entre idade e sucesso terapêutico. Conclu- \\
sões: Oestudo demonstrou que a adesão ao tratamento oclusivo desempe- \\
nha papel fundamental na eficácia terapêutica. Com isto, idade de início \\
do tratamento isoladamente não teve influência no sucesso terapêutico, \\
uma vez que foi possível obter boa adesão a despeito da idade. Além \\
disto, os resultados foram piores nos casos de ambliopia grave, nos quais \\
a adesão foi menor.
\end{tabular}

Descritores: Ambliopia/terapia; Cooperação do paciente; Curativos oclusivos; Eficácia; Estrabismo; Acuidade visual; Hospitais universitários; Estudos retrospectivos

\section{INTRODUÇÃO}

Para que haja desenvolvimento visual normal, é necessário que o cérebro receba sinais provenientes de imagens retinianas nítidas das fóveas dos dois olhos simultaneamente.

O termo ambliopia vem do grego (amblys: borrado, ops: olho) e significa visão borrada. E pode ser definido como uma baixa de acuidade visual uni ou bilateral causada por falta de estímulo adequado à retina durante $\mathrm{o}$ período crítico de desenvolvimento das funções visuais, o que pode causar lesões neurológicas secundárias representadas por atrofia de neurônios do corpo geniculado lateral e córtex estriado ${ }^{(1-2)}$. Apresenta como principais causas: estrabismo, altas ametropias não corrigidas, anisometropias e opacidades de meios. 
A severidade da ambliopia depende de vários fatores incluindo a época de aparecimento do estímulo inadequado, tempo de exposição a esse estímulo e a severidade do borramento da imagem.

Quanto mais precoce o início do tratamento, melhor é o prognóstico de reversão da ambliopia, apesar de terem sido demonstrados bons índices de cura em crianças que iniciaram tardiamente o tratamento ${ }^{(3)}$. Isso indica que haveria plasticidade neural para reverter as alterações causadas pela privação visual no corpo geniculado lateral e córtex visual ${ }^{(4-5)}$.

O tratamento de escolha não mudou nos últimos dois séculos e consiste na oclusão do olho bom, apesar dos relatos de sucesso variarem entre 30 a $92 \%(6-7)$.

O objetivo deste estudo foi avaliar a influência da adesão ao tratamento, da gravidade da ambliopia e da idade de início do tratamento no resultado visual final obtido em pacientes com ambliopia por estrabismo submetidos à terapia oclusiva no Setor de Motilidade Ocular Extrínseca do Departamento de Oftalmologia do Hospital das Clínicas da Faculdade de Medicina Universidade de São Paulo (HCFMUSP).

\section{MÉTODOS}

Foi realizado um estudo retrospectivo através da análise de 569 prontuários de pacientes com diagnóstico de ambliopia por estrabismo que foram acompanhados no Setor de Motilidade Ocular Extrínseca do Departamento de Oftalmologia do HCFMUSP no período 1983 a 2000.

Todos os pacientes foram submetidos a exame oftalmológico completo com os seguintes itens:

a) Medida de acuidade visual com tabela de Snellen nos pacientes que informavam acuidade visual, ou verificação do olhar preferencial ("Teller") para aqueles incapazes de informar acuidade visual. Quando não foi possível obter a acuidade visual inicial na primeira consulta, foi utilizada a primeira medida de visão que o paciente informou.

b) Refração sob cicloplegia (com ciclopentolato 1\%).

c) Biomicroscopia.

d) Fundoscopia.

e) Avaliação da motilidade ocular: teste de cobertura simples e alternada; teste de cobertura alternada com prisma ou teste de Krimsky (em pacientes incapazes de fixar alvo acomodativo).

f) Avaliação sensorial com estereoacuidade ("Titmus stereotest").

Foram incluídos no estudo apenas os pacientes com idade entre 0 e 12 anos na data da primeira consulta e que compareceram aos retornos de forma regular. O tempo de seguimento mínimo foi de 6 meses. Foram excluídos os pacientes que tiveram perda de seguimento por não retornarem às consultas, pacientes com nistagmo ou com doenças oculares associadas como: glaucoma congênito, cicatrizes de coriorretinite macular e pacientes com idade superior a 12 anos na $1^{\underline{a}}$ consulta.

Os pacientes selecionados foram classificados quanto ao sexo, idade no início do seguimento, gravidade da ambliopia, tipo de estrabismo, adesão ao tratamento e resultado visual final obtido.

A idade foi analisada por faixa etária, sendo subdividida em 4 grupos:

I) 0 a 2 anos e 11 meses;

II) 3 a 4 anos e 11 meses;

III) 5 a 6 anos e 11 meses;

IV) 7 a 12 anos.

Os pacientes foram classificados quanto ao tipo de estrabismo: microtropia, esotropia congênita ou infantil (aparecimento no primeiro ano de vida), esotropia totalmente acomodativa, esotropia parcialmente acomodativa, exotropia, hipertropia que podiam estar associados ou não a anisometropia.

A ambliopia foi considerada leve quando a acuidade visual no pior olho era maior que 0,5 até 0,8 , moderada quando a acuidade visual estava entre 0,5 e 0,3 e grave quando a acuidade visual era menor que 0,3 .

A adesão à terapia oclusiva foi considerada boa quando o paciente fazia uso correto da oclusão durante todo o período de acompanhamento ou pelo menos a partir da $3^{\underline{a}}$ consulta até concluir o tratamento. Aqueles pacientes que não usaram a oclusão corretamente após dois ou mais controles consecutivos após a $1^{\underline{a}}$ consulta ou não mantiveram a constância no decorrer do período de acompanhamento foram considerados como tendo má adesão ao tratamento. As informações relativas à adesão foram baseadas nas anotações de prontuário que foram feitas de maneira detalhada pelos ortoptistas.

O resultado do tratamento foi classificado em 4 estágios diferentes de acordo com a acuidade visual final:

1) Sucesso: quando a acuidade visual final atingiu índices entre 1,0 e 0,8 com melhora de pelo menos uma linha na tabela de Snellen.

2) Melhora: para acuidade visual final entre 0,7 e $0,5 \mathrm{com}$ melhora de pelo menos uma linha na tabela de Snellen.

3) Melhora parcial: para acuidade visual final entre 0,4 e 0,2 com melhora de pelo menos uma linha na tabela de Snellen.

4) Insucesso: quando a acuidade visual final permaneceu menor que 0,2 .

Os resultados foram submetidos à análise estatística através do teste de Fisher.

\section{RESULTADOS}

Foram avaliados 569 prontuários de pacientes dos quais 198 $(34,8 \%)$ puderam ser utilizados de acordo com os critérios de inclusão estabelecidos. Foram excluídos 152 pacientes $(26,7 \%)$ que perderam o seguimento por não comparecerem às consultas, 198 pacientes $(34,8 \%)$ que tinham idade superior a 12 anos na primeira consulta e 3 pacientes $(0,5 \%)$ por apresentarem nistagmo. Além disso, 18 pacientes $(3,2 \%)$ foram excluídos por serem portadores de outras doenças oculares associadas: 3 pacientes com glaucoma congênito, 6 pacientes com catarata congênita, 8 pacientes com cicatriz de coriorretinite macular e 1 paciente com leucoma corneano secundário a úlcera de córnea. 
Do total de 198 pacientes selecionados, 100 eram do sexo feminino (50,5\%) e 98 eram do sexo masculino (49,5\%).

O grupo selecionado apresentava os seguintes diagnósticos: 96 pacientes $(48,5 \%)$ com esotropia congênita ou infantil, 62 pacientes $(31,3 \%)$ com esotropia parcialmente acomodativa, 19 pacientes $(9,6 \%)$ com exotropia, 9 pacientes $(4,5 \%)$ com microtropia, 5 pacientes $(2,5 \%)$ com esotropia totalmente acomodativa e 2 pacientes $(1,0 \%)$ com síndrome de Duane.

Associadas às doenças acima, observamos 8 pacientes $(4,0 \%)$ com hipertropia, 18 pacientes $(9,1 \%)$ com anisometropia, e 3 pacientes $(1,5 \%)$ com ptose.

Com relação à distribuição dos pacientes por faixas etárias, 10 pacientes $(5,0 \%)$ pertenciam ao grupo I, 39 pacientes $(19,7 \%)$ pertenciam ao grupo II, 54 pacientes $(27,3 \%)$ pertenciam ao grupo III e 95 pacientes $(48,0 \%)$ pertenciam ao grupo IV.

Quanto à gravidade da ambliopia, 36 pacientes $(18,2 \%)$ apresentavam ambliopia leve, 48 (24,2\%) apresentavam ambliopia moderada e $114(57,6 \%)$ apresentavam ambliopia grave. A ambliopia grave foi predominante nos 4 grupos etários estudados, como se observa na tabela 1 .

\begin{tabular}{|lcrrr|}
\hline \multicolumn{4}{|c|}{ Tabela 1. Gravidade da ambliopia nos diferentes grupos etários } \\
& \multicolumn{1}{c}{ Leve } & \multicolumn{1}{c|}{ Moderada } & \multicolumn{1}{c|}{ Grave } \\
Grupo I $n=10$ & $1(10,0 \%)$ & $3(30,0 \%)$ & $6(60,0 \%)$ \\
Grupo II $n=39$ & $7(18,0 \%)$ & $11(28,2 \%)$ & $21(53,8 \%)$ \\
Grupo III $n=54$ & $7(13,0 \%)$ & $13(24,0 \%)$ & $34(63,0 \%)$ \\
Grupo IV $n=95$ & $21(22,1 \%)$ & $21(22,1 \%)$ & $53(55,8 \%)$ \\
\hline
\end{tabular}

A adesão ao tratamento oclusivo foi boa em 118 pacientes $(59,6 \%)$ e má em 80 pacientes $(40,4 \%)$. Os resultados, considerando-se a adesão ao tratamento, estão expressos na tabela 2. A análise estatística demonstrou que a grande maioria dos pacientes que tiveram boa adesão obtiveram sucesso ou pelo menos algum grau de melhora, enquanto a maioria dos pacientes com má adesão demonstraram insucesso ao tratamento $(\mathrm{p}=0,001)$.

Não houve diferença estatística entre a adesão nos diferentes grupos etários: 60,0\% de boa adesão no grupo I, 59,0\% no grupo II, $58,1 \%$ no grupo III e $58,8 \%$ no grupo IV. No total de pacientes estudados a taxa de sucesso foi $39,9 \%, 17,2 \%$ de melhora da acuidade visual, $10,1 \%$ de melhora parcial e $32,8 \%$ de insucesso.

Os resultados do tratamento oclusivo de acordo com gravidade da ambliopia podem ser observados na tabelas 3,4 e 5 .

Os dados das tabelas 3,4 e 5 foram submetidos a análise estatística que mostrou haver uma relação direta entre a aderência e o percentual de sucesso nos casos de ambliopia moderada $(\mathrm{p}=0,035)$ e de ambliopia grave $(\mathrm{p}=0,001)$, não tendo sido observada essa relação nos casos de ambliopia leve $(\mathrm{p}=0,215)$. Além disso, observou-se relação inversamente proporcional entre a aderência e a gravidade da ambliopia, isto é, quanto mais grave a ambliopia, menor foi a aderência $(\mathrm{p}=0,001)$.

Os resultados do tratamento oclusivo de acordo com os grupos etários podem ser observados na tabela 6 .

A análise estatística da tabela 6 não demonstrou melhores resultados diretamente proporcionais a grupos etários mais

\begin{tabular}{|lrrrrr|}
\hline \multicolumn{7}{c}{ Tabela 2. Resultado do tratamento de acordo com a adesão } \\
Boa adesão & $118(59,6 \%)$ & Sucesso & Melhora & Melhora parcial & Insucesso \\
Má adesão & $80(40,4 \%)$ & $60(50,8 \%)$ & $29(24,6 \%)$ & $10(8,5 \%)$ & $19(16,1 \%)$ \\
\hline
\end{tabular}

\begin{tabular}{|lccccc|}
\hline \multicolumn{7}{|c|}{ Tabela 3. Resultado do tratamento de acordo com a adesão nos pacientes com ambliopia leve } \\
Ambliopia leve & \multicolumn{7}{c}{ Sucesso } & Melhora & Melhora parcial & Insucesso \\
Boa adesão & $26(72,2 \%)$ & $21(80,8 \%)$ & $2(7,7 \%)$ & 0 & $3(11,5 \%)$ \\
Má adesão & $10(27,8 \%)$ & $6(60,0 \%)$ & 0 & 0 & $4(40 \%)$ \\
\hline
\end{tabular}

\begin{tabular}{|lccccc|}
\hline \multicolumn{7}{c}{ Tabela 4. Resultado do tratamento de acordo com a aderência nos pacientes com ambliopia moderada } \\
\hline Ambliopia moderada & Sucesso & Melhora & Melhora parcial & Insucesso \\
Boa adesão & $37(77,0 \%)$ & $22(59,5 \%)$ & $10(27 \%)$ & $1(2,7 \%)$ & $4(10,8 \%)$ \\
Má adesão & $11(23,0 \%)$ & $4(36,4 \%)$ & $1(9,1 \%)$ & $2(18,1 \%)$ & $4(36,4 \%)$ \\
\hline
\end{tabular}

\begin{tabular}{|c|c|c|c|c|c|}
\hline \multicolumn{2}{|c|}{ Ambliopia grave } & \multirow{2}{*}{$\begin{array}{l}\text { Sucesso } \\
17(30,9 \%)\end{array}$} & \multirow{2}{*}{$\begin{array}{c}\text { Melhora } \\
17(30,9 \%)\end{array}$} & \multirow{2}{*}{$\begin{array}{c}\text { Melhora parcial } \\
9(16,4 \%)\end{array}$} & \multirow{2}{*}{$\begin{array}{l}\text { Insucesso } \\
12(21,8 \%)\end{array}$} \\
\hline Boa adesãc & $55(48,2 \%)$ & & & & \\
\hline Má adesão & $59(51,8 \%)$ & $9(15,3 \%)$ & $4(6,8 \%)$ & $8(13,5 \%)$ & $38(64,4 \%)$ \\
\hline
\end{tabular}




\begin{tabular}{|c|c|c|c|c|}
\hline & Sucesso & Melhora & Melhora parcial & Insucesso \\
\hline Grupo I $n=10$ & $7(70,0 \%)$ & $1(10,0 \%)$ & $1(10,0 \%)$ & $1(10,0 \%)$ \\
\hline Grupo II n=39 & $18(46,2 \%)$ & $6(15,4 \%)$ & $2(5,1 \%)$ & $13(33,3 \%)$ \\
\hline Grupo III n=54 & $21(38,9 \%)$ & $4(7,4 \%)$ & $7(13,0 \%)$ & $22(40,7 \%)$ \\
\hline Grupo IV n=95 & $33(34,7 \%)$ & $23(24,2 \%)$ & $10(10,5 \%)$ & $29(30,5 \%)$ \\
\hline
\end{tabular}

jovens, isto é, não foi possível estabelecer associação entre sucesso e idade dos pacientes $(\mathrm{p}=0,107)$.

\section{DISCUSSÃO}

Existem alguns fatores que limitam a avaliação da eficácia do tratamento da ambliopia, como a dificuldade de medida de acuidade visual em crianças e a freqüente perda de pacientes por abandono de tratamento $^{(8)}$, fato esse observado nesse estudo, onde houve $26,7 \%$ de perda de seguimento, motivo pelo qual optou-se por excluir esses pacientes da análise final dos resultados.

É muito freqüente a chegada de pacientes amblíopes tardiamente em nosso serviço, o que foi demonstrado pela alta taxa de pacientes excluídos do estudo por terem mais de 12 anos $(34,8 \%)$. Mesmo entre os pacientes incluídos, houve predominância das faixas etárias mais avançadas (grupos III e IV). Apesar disso, não foi possível demonstrar estatisticamente, como seria esperado, a obtenção de melhores resultados nas menores faixas etárias, pois observou-se resultados satisfatórios em todos os grupos etários estudados, fato já demonstrado em outros estudos ${ }^{(4-5)}$. Portanto, o estudo sugere que a idade de início do tratamento não tem influência no sucesso terapêutico, demonstrando que o tratamento oclusivo deve ser instituído em qualquer dos grupos etários.

Embora existam outros estudos conflitantes em relação a esse dado $^{(8-10)}$, não houve diferença estatística entre a adesão nos diversos grupos etários o que é semelhante ao estudo de alguns autores ${ }^{(11)}$.

Notamos que a adesão teve um papel fundamental nos resultados do tratamento, pois de acordo com a análise estatística da tabela 2, a boa adesão demonstrou estar relacionada com altas taxas de sucesso enquanto que a má adesão relaciona-se com altas taxas de insucesso $(\mathrm{p}=0,001)$.

Quando se analisou o papel da adesão de cada grupo isolado de ambliopia leve, moderada e grave, apenas nos casos de ambliopia leve não foi possível estabelecer relação estatística diretamente proporcional entre boa aderência e melhores resultados $(\mathrm{p}=0,215)$ talvez devido ao pequeno número de pacientes com ambliopia leve obtido em nossa amostra. A adesão ao tratamento foi maior nos grupos de ambliopia leve e moderada, obtendo-se sucesso na maioria dos pacientes. Nos casos de ambliopia grave, que foram os mais freqüentes em nossa amostra independente da faixa etária, observamos menor adesão, com menor porcentagem de sucesso $(\mathrm{p}=0,001)$, dado também obtido por outros autores ${ }^{(10)}$.

\section{CONCLUSÕES}

O tratamento da ambliopia fica impossibilitado na maioria das vezes tanto pela chegada tardia dos pacientes para o primeiro atendimento (após os 12 anos de idade), quando o problema já pode ser irreversível, quanto pelo freqüente abandono do tratamento.

O estudo demonstrou que a adesão ao tratamento oclusivo desempenha um papel fundamental na eficácia terapêutica. A idade de início do tratamento isoladamente não teve influência no sucesso terapêutico, uma vez que foi possível obter boa adesão a despeito da idade. Além disto, a adesão ao tratamento foi menor nos casos de ambliopia mais grave, com conseqüente piora dos resultados.

\section{ABSTRACT}

Purpose: To evaluate the influence of compliance with treatment, severity of amblyopia and age at the beginning of treatment in patients with strabismic amblyopia submitted to patching. Methods: The data were selected from medical records of 569 patients seen at the Department of Ophthalmology, University of São Paulo during the period of 1983 to 2000. Exclusion criteria: loss of follow-up, age over 12 years, presence of nystagmus and other ocular diseases. The patients were divided into four groups according to age and classified based on the type of strabismus, compliance with treatment and severity of amblyopia. Statistical analysis was performed using Fisher's test. Results: 198 (34.8\%) patients were enrolled in the study. There was no difference regarding compliance among the age groups. The best success rate was obtained when there was good compliance with the treatment, independent of the degree of amblyopia. But worse compliance was observed among the patients with more severe amblyopia, who were the majority of patients of the study, and who showed the worst success rate. The success rate was not related to the patients' age. Conclusion: The study showed that compliance with patching plays a fundamental role in therapeutic efficacy. Thus, age at the beginning of treatment alone did not influence the success rate since it was possible to obtain good compliance independent of age. In addition, worse results were associated with severe amblyopia and less compliance.

Keywords: Amblyopia/therapy; Patient compliance; Occlusive dressings; Efficacy; Strabismus; Visual acuity; Hospitals, university; Retrospective studies 


\section{REFERÊNCIAS}

1. Hendrickson AE, Movshon JÁ, Eggers HM, Gizzi MS, Boothe RG, Kiorpes L. Effects of early unilateral blur on the macaque's visual system. II. Anatomical observations. J Neurosci. 1987;7(5):1327-39.

2. Von Noorden GK, Crawford MLJ, Levacy RA. The lateral geniculate nucleus in human anisometropic amblyopia. Invest Ophthalmol Vis Sci. 1983;24(6):788-90.

3. Ellis FD, Schlaegel TF. Unexpected visual recovery: organic amblyopia? Am Orthopt J. 1991;31:7

4. Campos E. Amblyopia. Surv Ophthalmol. 1995;40(1):23-39.

5. Salata ACF, Villaça VTN, Roma RL, Norato DYJ, Carvalho KMM. Terapia oclusiva em ambliopia: fatores prognósticos. Arq Bras Oftalmol. 2001;64(2):123-6.

6. Hiscox F, Strong N, Thompson JR, Minshull C, Woodruff G. Occlusion for amblyopia: a comprehensive survey of outcome. Eye 1992;6(Pt 3):300-4.
7. Monteiro de Carvalho KM, Simões MST, Sanabria LBG, Kara-José N. Ambliopia: necessidade de prevenção pré-escolar [resumo]. Arq Bras Oftalmol. 1996;59(Supl):354.

8. Smith LK, Thompson JR, Woodruff G, Hiscox F. Factors affecting treatment compliance in amblyopia. J Pediatr Ophthalmol Strabismus. 1995;32(2):98-101. Comment in: J Pediatr Ophthalmol Strabismus. 1995;32(6):399-400.

9. Souza EC, Carvalho AKR, Pulchinelli A, Gass C, Rothe E, Vanzella LL, et al. Cura e recorrência da ambliopia após terapia oclusiva. Arq Bras Oftalmol. 1994;57(1):16-9.

10. Nucci P, Alfarano R, Piantadina A, Brancato R. Compliance in antiamblyopia occlusion therapy. Acta Ophthalmol (Copenh). 1992;70(1):128-31.

11. Oliver M, Neumann R, Chaimovich Y, Gotesman N, Shimshoni M. Compliance and results of treatment for amblyopia in children more than 8 years old. Am J Ophthalmol. 1986;102(3):340-5.

\section{Simpósio Internacional de Córnea e Lentes de Confato - SINCLEC VI Curso Infernacional de Claucoma II Simpósio da Sociedade Ibero-Americana de Claucoma}

\section{9 e 20 de Maio de 2006}

\section{Instifuto de Ensine e Pescuisc de Hospilal Sírio hỉman̂̀s}

\section{Sã॰ Paulo - SP}

INFORMAÇÕES: JDE Comunicação e Eventos

Tels.: (11) 5084-5284/5084-9174/5082-3030

Fax: (11) 5574-8261

E-mail: jdecomev@uol.com.br 